\title{
Coherent population trapping in negatively charged self-assembled quantum dots using a train of femtosecond pulses
}

$\operatorname{AUTHOR}(S)$ :

Kumar, Parvendra; Nakajima, Takashi

\section{CITATION:}

Kumar, Parvendra ... [et al]. Coherent population trapping in negatively charged selfassembled quantum dots using a train of femtosecond pulses. Physical Review A 2015, 91(2): 023832.

\section{ISSUE DATE:}

2015-02

URL:

http://hdl.handle.net/2433/196632

RIGHT:

(C)2015 American Physical Society 


\title{
Coherent population trapping in negatively charged self-assembled quantum dots using a train of femtosecond pulses
}

\author{
Parvendra Kumar ${ }^{*}$ and Takashi Nakajima ${ }^{\dagger}$ \\ Institute of Advanced Energy, Kyoto University, Gokasho, Uji, Kyoto 611-0011, Japan \\ (Received 16 November 2014; published 26 February 2015)
}

\begin{abstract}
We demonstrate the coherent population trapping in a single quantum dot and an ensemble of negatively charged quantum dots using a train of femtosecond pulses. Particularly in an ensemble of quantum dots, we show that the detrimental effects due to the inhomogeneous distribution of their properties can be minimized by appropriately choosing the pulse-train parameters and the magnetic-field strength in such a way that the electron-Zeeman splitting is an integer multiple of the pulse repetition rate.
\end{abstract}

DOI: 10.1103/PhysRevA.91.023832

PACS number(s): 42.50.Gy, 42.50.Ar, 73.21.La

\section{INTRODUCTION}

If a quantum system is coherently driven into the so-called dark state which does not interact with the radiation, it becomes transparent to the radiation. Such laser-induced transparency is termed electromagnetically induced transparency (EIT) [1], which has been intensively studied in atoms and molecules for various applications, such as slow light [2,3], quantum information storage [4], refractive index enhancement [5], four- and six-wave mixing [6], and quantum repeaters [7]. The accumulating population in the dark state during the interaction with coherent radiation cannot be transferred to anywhere else due to its immunity to the radiation, and coherent population trapping (CPT) [8] takes place. Namely, EIT is always accompanied by CPT and vice versa. Although most of the studies on EIT and CPT employ the continuous-wave lasers or relatively long single laser pulses, the same phenomena also occur by the use of a pulse train. Indeed, Kocharovskaya and Khanin have demonstrated CPT in a three-level $\Lambda$-like system using a train of ultrafast pulses [9], which is followed by other related works using a pulse train [10-15].

Recently, substantial attention has been paid towards the realization of EIT [16-18] and CPT [19-21] in quantum dots (QDs) instead of atoms and molecules. With a negatively charged single QD, CPT of an electron spin has been realized using continuous-wave lasers [19]. In their study, a strong magnetic field $(2.64 \mathrm{~T})$ is required to realize a three-level $\Lambda$-like system out of a four-level system. For some applications of EIT and CPT, however, the use of an ensemble of QDs is more desirable. The essential difference between an ensemble of atoms and molecules and that of QDs is that for the former what matters is the inhomogeneity in effective transition frequencies due to the Doppler broadening, whereas for the latter what matters is the inhomogeneity in terms of dipole moments, trion transition frequencies, and electron-spin $g$ factors. As a consequence the degree of achievable coherence in an ensemble of QDs can be limited [22], and here we report on CPT in an ensemble of QDs. We should point out, however, that a novel scheme has recently been proposed and demonstrated by Greilich and co-workers [23-25] to realize a single-mode precession of electron spins in an

\footnotetext{
*parvendra1986@gmail.com

†nakajima@iae.kyoto-u.ac.jp
}

ensemble of singly charged QDs by suppressing the influence of inhomogeneous broadening of electron-spin $g$ factors by using a picosecond pulse train under the presence of a magnetic field. The essence of their idea is to modify the continuous density of electron-spin precession modes in an ensemble of QDs into a discrete (comblike) density by the irradiation of a train of short laser pulses to induce a burst of electron-spin polarization with the repetition time of pulses, whereas the QDs which do not satisfy the above mode-locking condition are eventually driven into the synchronized mode due to the fluctuation of the electron-spin precession frequencies in the collective hyperfine field of the dot nuclei.

In this paper, we theoretically demonstrate CPT in a single QD and an ensemble of negatively charged QDs using a pulse train. In particular we show that the undesired inhomogeneous properties in an ensemble of QDs can be minimized if the pulse-train parameters and the magnetic-field strength are appropriately chosen. Related to our paper Shabaev et al. have undertaken a theoretical study to realize slow light in an ensemble of QDs using a pulse train [26]. In contrast to their work, the main focus of our paper is to show how maximum coherence can be prepared using a train of femtosecond pulses. For this purpose we take into account the inhomogeneous properties of an ensemble of QDs in terms of dipole moments, trion transition frequencies, and electron-spin $g$ factors, and carry out through numerical calculations to demonstrate that the appropriate choice of the parameters for the pulse train and the magnetic-field strength leads to the creation of maximum coherence even in an ensemble of QDs.

\section{THEORY}

We consider the negatively charged self-assembled InAs QDs with the growth direction along the $z$ axis. The magnetic field is applied along the $x$ axis (Voigt geometry). In Fig. 1, we depict the four-level QD system and the corresponding selection rules. Here, $|X \pm\rangle=2^{-1 / 2}(|\downarrow\rangle \pm|\uparrow\rangle)$ represent the two electron ground states with spins polarized along the $x$ axis, where $|\uparrow\rangle$ and $|\downarrow\rangle$ denote the electron spins aligned along the $z$ axis and $|T \pm\rangle=2^{-1}(|\uparrow \downarrow\rangle)(|\Downarrow\rangle \pm|\uparrow\rangle)$ represent the trion states polarized along the $x$ axis, where $|\Downarrow\rangle=$ $|3 / 2,-3 / 2\rangle$ and $|\Uparrow\rangle=|3 / 2,3 / 2\rangle$ denote heavy-hole states aligned along the $z$ axis. Under the externally applied magnetic field in the Voigt geometry, the electron spin and trion states 


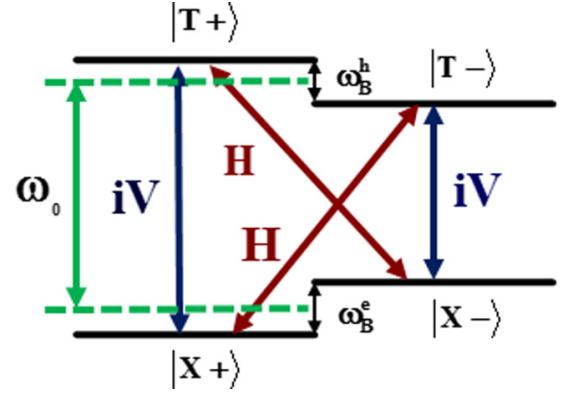

FIG. 1. (Color online) Four-level model of a negatively charged quantum dot.

are split by the Zeeman frequencies; $\omega_{B}^{e}=\left|g_{x}^{e} \mu_{B} B_{x}\right| / h$ and $\omega_{B}^{h}=\left|g_{x}^{h} \mu_{B} B_{x}\right| / h$, respectively, where $g_{x}^{e}$ and $g_{x}^{h}$ are the electron and heavy-hole spin $g$ factors, $\mu_{B}$ is the Bohr magneton, $h$ is the Planck constant, and $B_{x}$ is the magnitude of the applied magnetic field. The linearly polarized transitions $|X \pm\rangle \leftrightarrow|T \pm\rangle$ are labeled as $V$ and $H$. These transitions are referred to as vertically $(V)$ and horizontally $(H)$ polarized with respect to the polarization axis $(\vec{\eta})$ of QDs $V \perp \vec{\eta}$ and $H \| \vec{\eta}$. In the present study, we ignore the effect induced by the heavy- and light-hole mixings since this only rotates the polarization axis and has no influence on the polarization selection rules [27].

The electric field of a circularly polarized pulse train can be written as [28] $E(t)=\sum_{n=0}^{N-1} \frac{E_{0}}{\sqrt{2}} f\left(t-n T_{R}\right)$ $\left\{\cos \left[\omega_{c}\left(t-n T_{R}\right)+i \Delta \phi\right] \widehat{x}+\sin \left[\omega_{c}\left(t-n T_{R}\right)+i \Delta \phi\right] \widehat{y}\right\}$, where $f\left(t-n T_{R}\right)=\exp \left\{-\left[\left(t-n T_{R}\right) / \tau_{p}\right]^{2}\right\}$ is the pulse envelope, $N$ is the number of pulses in the pulse train, $E_{0}$ is the peak value of the electric-field amplitude, $\omega_{c}$ is the central frequency, $T_{R}$ is the pulse repetition time, $\tau_{p}$ is related to the pulse duration through $\tau_{\mathrm{FWHM}}=1.177 \tau_{p}, \Delta \phi$ is the phase difference between two consecutive pulses, and $\widehat{x}, \widehat{y}$ are the unit vectors.
The interaction between the pulse train and the four-level QD system is described by the following Hamiltonian written in the basis of $|X+\rangle,|X-\rangle,|T-\rangle$, and $|T+\rangle$. It reads

$$
H=\hbar\left(\begin{array}{cccc}
\omega_{X+} & 0 & -\Omega_{H}(t) & -\Omega_{V}(t) \\
0 & \omega_{X-} & -\Omega_{V}(t) & -\Omega_{H}(t) \\
-\Omega_{H}^{*}(t) & -\Omega_{V}^{*}(t) & \omega_{T-} & 0 \\
-\Omega_{V}^{*}(t) & -\Omega_{H}^{*}(t) & 0 & \omega_{T+}
\end{array}\right)
$$

Here,

$$
\Omega_{H}(t)=\frac{\mu_{x} E_{0}}{\sqrt{2} \hbar} \sum_{n=0}^{N-1} f\left(t-n T_{R}\right) \cos \left[\omega_{c}\left(t-n T_{R}\right)+i \Delta \phi\right]
$$

and

$$
\Omega_{V}(t)=\frac{i \mu_{y} E_{0}}{\sqrt{2} \hbar} \sum_{n=0}^{N-1} f\left(t-n T_{R}\right) \sin \left[\omega_{c}\left(t-n T_{R}\right)+i \Delta \phi\right]
$$

are the time-dependent Rabi frequencies with the associated transition dipole moments $\mu_{x}$ and $\mu_{y}$ where $\mu_{x}=\mu_{T+X-}=$ $\mu_{T-X+}$ and $\mu_{x}^{*}=\mu_{x}$ whereas $\mu_{y}=\mu_{T+X+}=\mu_{T-X-}, \mu_{y}^{*}=$ $-\mu_{y}$, and $\mu_{y}=i \mu_{x}$ [29]. The area of the individual pulse in the pulse train is defined as $\Theta=\int_{-\infty}^{\infty} \alpha(t) d t$, with

$$
\alpha(t)=\mu_{x} E_{0} f(t) / \sqrt{2} \hbar .
$$

With the Hamiltonian defined above the equation of motion of the four-level QD system shown in Fig. 1 can be described by the following master equation:

$$
\frac{d \rho}{d t}=-\frac{i}{\hbar}[H, \rho]+\mathcal{L}(\rho)
$$

where $\mathcal{L}(\rho)$ is the Linbladian operator that describes the relaxation and decoherence processes. It is given by

$$
\mathcal{L}(\rho)=\left(\begin{array}{ccccc}
\Gamma_{X+T-} \rho_{T-T-} & +\Gamma_{X+T+} \rho_{T+T+} & -\gamma_{X+X-} \rho_{X+X-} & -\gamma_{X+T-} \rho_{X+T-} & -\gamma_{X+T+} \rho_{X+T+} \\
-\gamma_{X-X+} \rho_{X-X+} & \Gamma_{X-T-} \rho_{T-T-} & +\Gamma_{X-T+} \rho_{T+T+} & -\gamma_{X-T-} \rho_{X-T-} & -\gamma_{X-T+} \rho_{X-T+} \\
-\gamma_{T-X+} \rho_{T-X+} & -\gamma_{T-X-} \rho_{T-X-} & -\Gamma_{X+T-} \rho_{T-T-} & -\Gamma_{X-T-} \rho_{T-T-} & -\gamma_{T-T+} \rho_{T-T+} \\
-\gamma_{T+X+} \rho_{T+X+} & -\gamma_{T+X-} \rho_{T+X-} & -\gamma_{T+T-} \rho_{T+T-} & -\Gamma_{X+T+} \rho_{T+T+} & -\Gamma_{X-T+} \rho_{T+T+}
\end{array}\right)
$$

in which $\Gamma_{n m}(n, m=X+, X-, T+, T-)$ is the decay rate from state $m$ to state $n$ and $\gamma_{n m}=\gamma_{m n}^{*}$ gives the damping rate of the coherence term $\rho_{n m}$. For simplicity we assume that $\Gamma_{X+T+}=\Gamma_{X+T-}=\Gamma_{X-T-}=\Gamma_{X-T+}=\Gamma$ and $\gamma_{T-X+}=$ $\gamma_{T-X-}=\gamma_{T+T-}=\gamma_{T+X-}=\gamma_{T+X+}=\gamma$. In the case of a single QD, we use the following typical parameters: $(\Gamma)^{-1}=0.855 \mathrm{~ns}$ [30], $(\gamma)^{-1}=0.3 \mathrm{~ns}$ [19], $\left(\gamma_{X-X+}\right)^{-1}=$ $10 \mu \mathrm{s}$ [31], and $B_{x}=1890 \mathrm{mT},\left|g_{x}^{e}\right|=0.378$ and $\left|g_{x}^{h}\right|=$ 0.202 [30], $\omega_{B}^{e}=10$ and $\omega_{B}^{h}=5.348 \mathrm{GHz}, \tau_{p}=100 \mathrm{fs}, \omega_{c}=$ $\omega_{T+X+}=\omega_{0}+\left(\omega_{B}^{e}+\omega_{B}^{h}\right) / 2, \omega_{0}=315 \mathrm{THz}$ and $\mu_{x}=8 \mathrm{D}$ [30], $\omega_{T+X-}=\omega_{T+X+}-\omega_{B}^{e}, \omega_{T-X+}=\omega_{T+X+}-\omega_{B}^{h}$, and $\omega_{T-X-}=\omega_{T+X+}-\omega_{B}^{e}-\omega_{B}^{h}$, where $\omega_{n m}=\omega_{n}-\omega_{m}$ and $\Delta \phi=0$. In the case of an ensemble of QDs, we choose the following typical parameters: $\left|\overline{g_{x}^{e}}\right|=0.57$ and $\left|\overline{g_{x}^{h}}\right|=0.81$ [25] are the mean values of electron- and heavy-hole spin $g$ factors, $\bar{\mu}_{x}=8 \mathrm{D}$ is the mean value of the dipole moment, $\left(\gamma_{X-X+}\right)^{-1}=3 \mu \mathrm{s}$ [32], $B_{x}=125.35 \mathrm{mT}, \omega_{B}^{e}=1$, and $\omega_{B}^{h}=$ $1.42 \mathrm{GHz}$ with all other parameters remaining to be the same with those for a single QD.

\section{RESULTS AND DISCUSSIONS}

We numerically solve Eq. (2) using a standard fourth-order Runge-Kutta method. Figure 2 depicts the accumulation of coherence in the single QD for different values of pulse intervals with the constant pulse area $\Theta=\pi / 20$ and the magnetic field $B_{x}=1890 \mathrm{mT}$. The accumulation of coherence for $T_{R}=$ $200 \mathrm{ps}$ is faster compared to those for $T_{R}=500 \mathrm{ps}, 2$, and $4 \mathrm{~ns}$. This is particularly due to the fact that for $T_{R}=200 \mathrm{ps}$ the QD is excited by nearly four pulses during the decay time of the trion states, which results in the faster accumulation of 


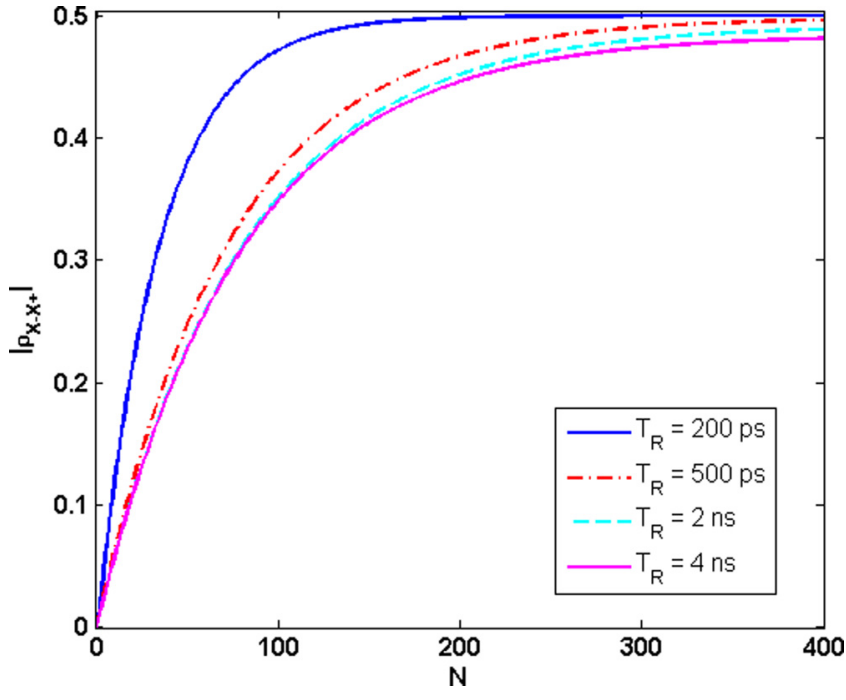

FIG. 2. (Color online) Accumulation of coherence in the single QD as a function of the irradiated number of pulses for the different values of pulse intervals.

coherence. In Fig. 2, for every chosen value of $T_{R}$, the so-called two-photon resonance condition $\omega_{B}^{e}=p f_{R}$ is satisfied where $p$ is an integer. We can see from Fig. 2 that the maximum and minimum values of coherence are 0.50 and 0.48 for $T_{R}=200 \mathrm{ps}$ and $T_{R}=4 \mathrm{~ns}$, respectively.

Next we study the feasibility of such pulse trains for the generation of maximum coherence with the increased values of pulse area. Figure 3(a) depicts the evolution of coherence for different values of pulse area at the constant pulse interval of $T_{R}=4$ ns. At $\Theta=\pi / 5$, maximum coherence $(\approx 0.5)$ is established after nearly 25 pulses, which means that coherent
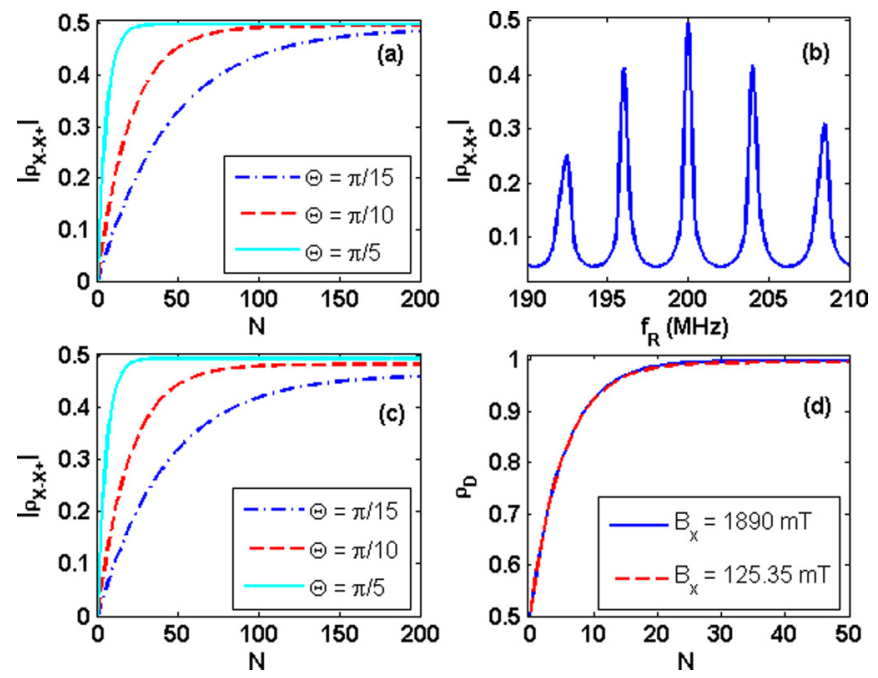

FIG. 3. (Color online) (a) Accumulation of coherence in the single QD for the different values of pulse area and (b) the corresponding evolution of coherence as a function of pulse repetition rate. (c) Accumulation of coherence in an ensemble of QDs for the different values of pulse area and (d) the corresponding evolution of dark state populations as a function of the irradiated number of pulses. electron spin can be generated within a much shorter time scale (100 ns) compared to the decoherence time $(10 \mu \mathrm{s})$ of QDs at the cryotemperature. This implies that the preparation of the coherent electron spin described in this paper may be applied for quantum information processing [20].

Next, in Fig. 3(b), we depict the final value of coherence for $N=50$ as a function of pulse repetition rate at the constant value of pulse area $\Theta=\pi / 5$ and magnetic field $B_{x}=1890 \mathrm{mT}$. We notice that coherence is maximum at $f_{R}=200 \mathrm{MHz}$ owing to the exact two-photon resonance condition. The occurrence of other peaks arises due to the two-photon near resonance. For the central peak in Fig. 3(b), the width of two-photon resonance (full width at half maximum) is $0.7 \mathrm{MHz}$. Therefore the width of two-photon resonance is very small compared to the electron-Zeeman frequency $\omega_{B}^{e}=10 \mathrm{GHz}$. Hence this scheme can be used for high-resolution spectroscopy of a single quantum dot. In Fig. 3(c), we show the accumulation of coherence in an ensemble of negatively charged QDs for $B_{x}=125.35 \mathrm{mT}$ while keeping the pulse interval $\left(T_{R}=5 \mathrm{~ns}\right)$ larger than the ensemble dephasing time of a few nanoseconds [25]. To calculate the dynamics of the ensemble of QDs we assume, for a moment, the identical values of dipole moments, trion transition frequencies, and electron-spin $g$ factor for all QDs. It can be seen that the maximum coherence $(\approx 0.5)$ can also be achieved in an ensemble of QDs by appropriately choosing the pulse-train parameters. In Fig. 3(d), we depict the dynamics of the dark state populations in a single QD and an ensemble of QDs at $B_{x}=1890 \mathrm{mT}$ and $B_{x}=125.35 \mathrm{mT}$, respectively. Bright and dark states in our four-level QD system can be written as $[13,33,34]|B\rangle=(|X+\rangle+|X-\rangle) / \sqrt{2}$ and $|D\rangle=(|X+\rangle-|X-\rangle) / \sqrt{2}$, respectively. Populations in these states are given by $\rho_{B}=\left(\rho_{X+X+}+\rho_{X-X_{-}}\right) / 2+\operatorname{Re}\left(\rho_{X-X+}\right)$ and $\rho_{D}=\left(\rho_{X+X+}+\rho_{X-X-}\right) / 2-\operatorname{Re}\left(\rho_{X-X+}\right)$. Initially, both states are equally populated, i.e., $\rho_{B}=0.5$ and $\rho_{D}=0.5$. Due to the accumulation of maximum coherence after the interaction with many pulses, nearly complete population is pumped to the dark state, and simultaneously the population in the bright state becomes zero (not shown). After the creation of the fully populated dark state, the pulses are no longer absorbed, and eventually EIT of the pulse train can take place.

Of course the assumptions we have made in Figs. 3(c) and 3(d) for the ensemble of QDs is too stringent, and in practice the ensemble of QDs suffers from the inhomogeneous distribution of dipole moments, trion transition frequencies, and electron-spin $g$ factors. To understand the effect of inhomogeneous distribution on the dark state population, we include them one by one. First, we study the effect of the inhomogeneous distribution of dipole moments among QDs. Assuming that the distribution function for the properties of an ensemble of QDs obeys the normal distribution the distribution function of dipole moments reads $f(\mu)=$ $\frac{1}{\sqrt{\pi} \alpha} \exp \left\{-\left[\left(\mu-\bar{\mu}_{x}\right) / \alpha\right]^{2}\right\}$. Following Ref. [35] the full width at half maximum (FWHM) of the distribution of dipole moments $\alpha_{\text {FWHM }}$ is assumed to be 1.88. In Fig. 4, we depict the variation in the dark state population in the single QD as a function of the value of dipole moments with $\bar{\mu}_{x}=8 \mathrm{D}$. We can see that the dark state population after irradiating the QD by $N=50$ is quite smaller than unity and significantly depends on the particular value of the dipole moment, especially for 


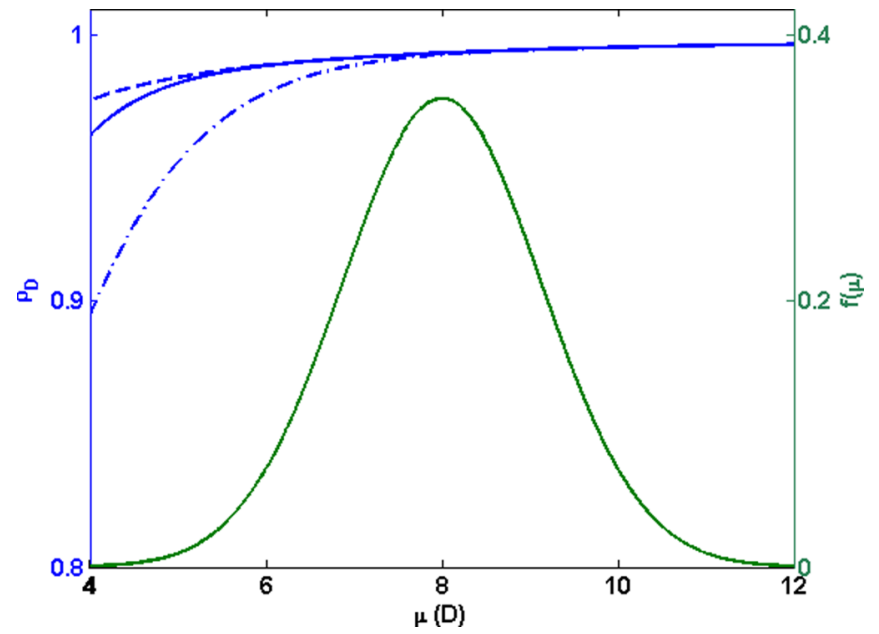

FIG. 4. (Color online) Variation in the dark state population in the single QD as a function of the value of dipole moments with $\bar{\mu}_{x}=8 \mathrm{D}, T_{R}=5 \mathrm{~ns}$, and $E_{0}=1.62 \times 10^{5} \mathrm{~V} / \mathrm{cm}$. The number of irradiated pulses are $N=50$ (dot-dashed line), 100 (solid line), and 200 (dashed line), respectively. The solid line at the center represents the distribution function $f(\mu)$.

the lower values of the dipole moments. However, after the excitation of the QD by 200 pulses, the dark state is fully populated $(\approx 98 \%)$ over the entire range of dipole moments.

This is particularly due to the pulse-to-pulse accumulation of coherence, which significantly nullifies the effect of variation in the dipole moments. The ensemble-averaged dark state population, $\bar{\rho}_{D}=\sum_{\mu} \frac{\rho_{D}(\mu) f(\mu)}{f(\mu)}$ for $N=50,100$, and 200 is found to be $99.09,99.29$, and $99.30 \%$, respectively. Therefore, by irradiating the QDs by 200 or more pulses, the effect of inhomogeneous distribution of the values of dipole moments can be minimized to the negligible level. Next, we study the effect of inhomogeneous distribution of trion transition frequencies. The FWHM of the inhomogeneous distribution in the trion transition frequencies is found to be $169 \mathrm{GHz}$ [36]. After repeating the same procedure with $T_{R}=5 \mathrm{~ns}, B_{x}=$ $125.35 \mathrm{mT}, N=200$, and $\Theta=\pi / 5$, the obtained value of the dark state population after the ensemble average is $99.52 \%$. Therefore the effect of inhomogeneous trion transition frequencies can also be minimized by appropriately choosing the pulse-train parameters and the magnetic-field strength. Finally we study the effect of the inhomogeneous distribution of electron-spin $g$ factors among QDs. We again assume that its distribution function obeys the normal distribution, i.e., $f(g)=\frac{1}{\sqrt{\pi} \beta} \exp \left\{-\left[\left(g-\overline{g_{x}^{e}}\right) / \beta\right]^{2}\right\}$. Following Ref. [24] we assume $\beta_{\mathrm{FWHM}}=0.004$.

Figure 5 depicts the variation in the dark state population in the single QD as a function of electron-spin $g$ factors after the irradiation of $N=200$ pulses with $\overline{g_{x}^{e}}=0.57$ under the different magnetic-field strengths. Here, we choose the values of magnetic field and pulse interval such that the condition $\overline{\omega_{B}^{e}}=p f_{R}$ is satisfied $\overline{\omega_{B}^{e}}=\left|\overline{g_{x}^{e}} \mu_{B} B_{x}\right| / h$. We can see that the effect of electron-spin $g$ factors becomes less pronounced for the smaller magnetic-field strength. This is particularly due to the fact that the broadening $\Delta \omega=\left|\beta_{\mathrm{FWHM}} \mu_{B} B_{x}\right| / h$ of the mode-locked Zeeman frequency $\overline{\omega_{B}^{e}}$ becomes smaller at the smaller values of magnetic fields [24]. The ensemble-averaged

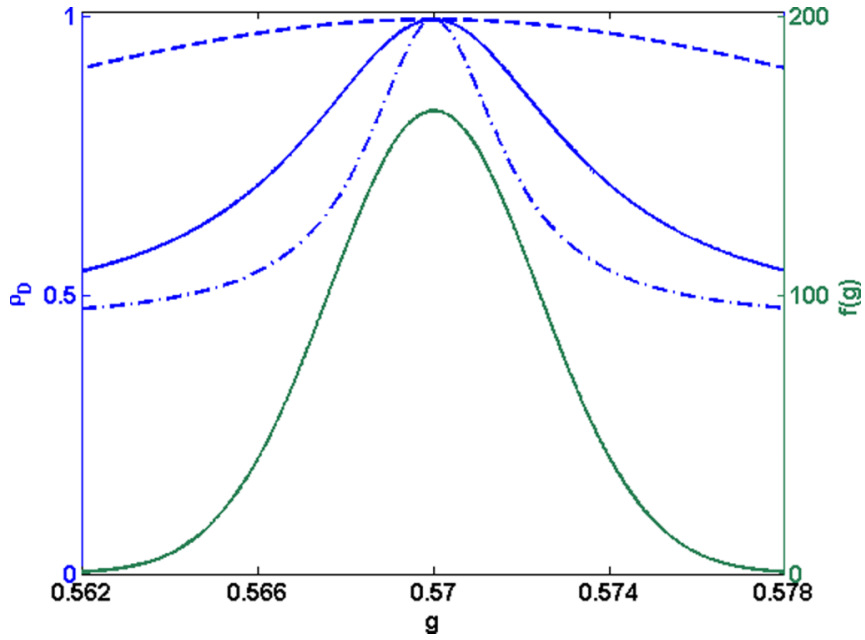

FIG. 5. (Color online) Variation in the dark state population in the single QD as a function of electron-spin $g$ factors with $\overline{g_{x}^{e}}=0.57$, $T_{R}=5 \mathrm{~ns}$, and $\Theta=\pi / 5$. The employed magnetic-field strengths are $B_{x}=250.70$ (dot-dashed line), 125.35 (solid line), and $25.07 \mathrm{mT}$ (dashed line), respectively. The solid line at the center represents the distribution function $f(g)$. The number of irradiated pulses is $N=200$.

dark state population for $B_{x}=250.70,125.35$, and $25.07 \mathrm{mT}$ is found to be $76.04 \%, 87.41 \%$, and $98.63 \%$, respectively. This also shows that the effect of inhomogeneous distribution of electron-spin $g$ factors can be minimized by appropriately choosing the pulse interval and the magnetic-field strength. Hence by appropriately choosing the pulse-train parameters and the magnetic-field strength, the effect of inhomogeneous distribution of QD properties, such as dipole moments, trion transition frequencies, and electron-spin $g$ factors can be minimized, which in turn lead to the creation of nearly maximum coherence. Maximum coherence prepared in this way can be exploited to transfer the information carried in the phase and amplitude of the input field to those of the output field through coherent Raman scattering via a light-hole trion state [37].

\section{CONCLUSIONS}

In conclusion we have demonstrated the coherent population trapping in a single QD as well as an ensemble of negatively charged QDs using a train of femtosecond pulses. In a single $\mathrm{QD}$, we have shown that the appropriate choice of pulse-train parameters enables us to achieve the maximum coherence in the two excitation regimes in which the pulse interval is shorter or longer than the decay time of the trion states. In particular nearly complete population transfer to the dark state or nearly complete population trapping can be realized if the electron-Zeeman frequency is chosen to be an integer multiple of the pulse repetition rate. Moreover, in the case of an ensemble of QDs, we have demonstrated that the effects of inhomogeneous distribution of QD properties can be minimized by appropriately choosing the pulse-train parameters and the magnetic-field strength. The coherence generated in an ensemble of QDs can be exploited to transfer information in the input field to that in the output field via 
coherent Raman scattering. This scheme can also be applied to realize the coherent population trapping in positively charged quantum dots.

\section{ACKNOWLEDGMENT}

This study was supported by a Grant-in-Aid for Scientific Research from the Ministry of Education and Science of Japan.
[1] S. E. Harris, Phys. Today 50(7), 36 (1997).

[2] D. Akamatsu, Y. Yokoi, M. Arikawa, S. Nagatsuka, T. Tanimura, A. Furusawa, and M. Kozuma, Phys. Rev. Lett. 99, 153602 (2007).

[3] C. Hang and G. Huang, Phys. Rev. A 77, 033830 (2008).

[4] C. Liu, Z. Dutton, C. H. Behroozi, and L. V. Hau, Nature (London) 409, 490 (2001).

[5] S. E. Harris, J. E. Field, and A. Imamoğlu, Phys. Rev. Lett. 64, 1107 (1990).

[6] Y. Zhang, A. W. Brown, and M. Xiao, Phys. Rev. Lett. 99, 123603 (2007).

[7] L.-M. Duan, M. D. Lukin, J. I. Cirac, and P. Zoller, Nature (London) 414, 413 (2001).

[8] M. O. Scully and M. S. Zubairy, Quantum Optics (Cambridge University Press, Cambridge, UK, 1997).

[9] O. A. Kocharovskaya and Y. I. Khanin, Sov. Phys. JETP 63, 945 (1986).

[10] D. Felinto, L. H. Acioli, and S. S. Vianna, Phys. Rev. A 70, 043403 (2004).

[11] A. A. Soares and L. E. E. de Araujo, Phys. Rev. A 76, 043818 (2007).

[12] A. A. Soares and L. E. E. de Araujo, J. Phys. B: At., Mol. Opt. Phys. 43, 085003 (2010).

[13] D. Aumiler, Phys. Rev. A 82, 055402 (2010).

[14] M. P. Moreno and S. S. Vianna, J. Opt. Soc. Am. B 28, 1124 (2011).

[15] G. Buica and T. Nakajima, Opt. Commun. 332, 59 (2014).

[16] S. Marcinkevičius, A. Gushterov, and J. P. Reithmaier, Appl. Phys. Lett. 92, 041113 (2008).

[17] J. Houmark, T. R. Nielsen, J. Mørk, and A.-P. Jauho, Phys. Rev. B 79, 115420 (2009).

[18] S. Michael, W. W. Chow, and H. C. Schneider, Phys. Rev. B 88, 125305 (2013).

[19] X. Xu, B. Sun, P. R. Berman, D. G. Steel, A. S. Bracker, D. Gammon, and L. J. Sham, Nat. Phys. 4, 692 (2008).

[20] D. Brunner, B. D. Gerardot, P. A. Dalgarno, G. Wüst, K. Karrai, N. G. Stoltz, P. M. Petroff, and R. J. Warburton, Science 325, 70 (2009).

[21] J. Houel, J. H. Prechtel, A. V. Kuhlmann, D. Brunner, C. E. Kuklewicz, B. D. Gerardot, N. G. Stoltz, P. M. Petroff, and R. J. Warburton, Phys. Rev. Lett. 112, 107401 (2014).
[22] K.-M. C. Fu, C. Santori, C. Stanley, M. C. Holland, and Y. Yamamoto, Phys. Rev. Lett. 95, 187405 (2005).

[23] A. Greilich, S. E. Economou, S. Spatzek, D. R. Yakovlev, D. Reuter, A. D. Wieck, T. L. Reinecke, and M. Bayer, Nat. Phys. 5, 262 (2009).

[24] A. Greilich, S. Spatzek, I. A. Yugova, I. A. Akimov, D. R. Yakovlev, A. L. Efros, D. Reuter, A. D. Wieck, and M. Bayer, Phys. Rev. B 79, 201305 (2009).

[25] A. Greilich, R. Oulton, E. A. Zhukov, I. A. Yugova, D. R. Yakovlev, M. Bayer, A. Shabaev, A. L. Efros, I. A. Merkulov, V. Stavarache, D. Reuter, and A. Wieck, Phys. Rev. Lett. 96, 227401 (2006).

[26] A. Shabaev, Z. Dutton, T. A. Kennedy, and A. L. Efros, Phys. Rev. A 82, 053823 (2010).

[27] X. Xu, Ph.D. thesis, The University of Michigan, 2008.

[28] S. T. Cundiff, J. Phys. D 35, R43 (2002).

[29] K. Truex, L. A. Webster, L.-M. Duan, L. J. Sham, and D. G. Steel, Phys. Rev. B 88, 195306 (2013).

[30] E. D. Kim, K. Truex, Y. Wu, A. Amo, X. Xu, D. G. Steel, A. S. Bracker, D. Gammon, and L. J. Sham, Appl. Phys. Lett. 97, 113110 (2010).

[31] J. R. Petta, A. C. Johnson, J. M. Taylor, E. A. Laird, A. Yacoby, M. D. Lukin, C. M. Marcus, M. P. Hanson, and A. C. Gossard, Science 309, 2180 (2005).

[32] A. Greilich, D. R. Yakovlev, A. Shabaev, A. L. Efros, I. A. Yugova, R. Oulton, V. Stavarache, D. Reuter, A. Wieck, and M. Bayer, Science 313, 341 (2006).

[33] T. Zanon, S. Guerandel, E. de Clercq, D. Holleville, N. Dimarcq, and A. Clairon, Phys. Rev. Lett. 94, 193002 (2005).

[34] E. A. Korsunsky and D. V. Kosachiov, Phys. Rev. A 60, 4996 (1999).

[35] A. Thränhardt, C. Ell, G. Khitrova, and H. M. Gibbs, Eur. Phys. J. B 27, 571 (2002).

[36] M. V. Gurudev Dutt, J. Cheng, Y. Wu, X. Xu, D. G. Steel, A. S. Bracker, D. Gammon, S. E. Economou, R.-B. Liu, and L. J. Sham, Phys. Rev. B 74, 125306 (2006).

[37] R. S. Bennink, A. M. Marino, V. Wong, R. W. Boyd, and C. R. Stroud, Jr., Phys. Rev. A 72, 023827 (2005). 